\title{
Die Bola: Von der Waffe zum selbstgebauten Sport- und Spielgerät
}

Spricht man von einer »Waffe» im historischen Sinn, so ist damit meistens ein Gegenstand gemeint, mit dem man Lebewesen erlegen oder wenigstens unschädlich machen kann. Waffen gibt es in vielerlei Ausprägungen: So gab es mehr oder weniger durchschlagkräftige, kleine und große Feuerwaffen und Waffen, die mit der eigenen Muskelkraft zur Wirkung gebracht werden müssen. Nur noch wenige der mit Muskelkraft betätigten Waffen werden heute weiterhin tatsächlich als Waffe eingesetzt, wobei die meisten davon allerdings zu Sport- und Spielgeräten umfunktioniert worden sind (z.B.

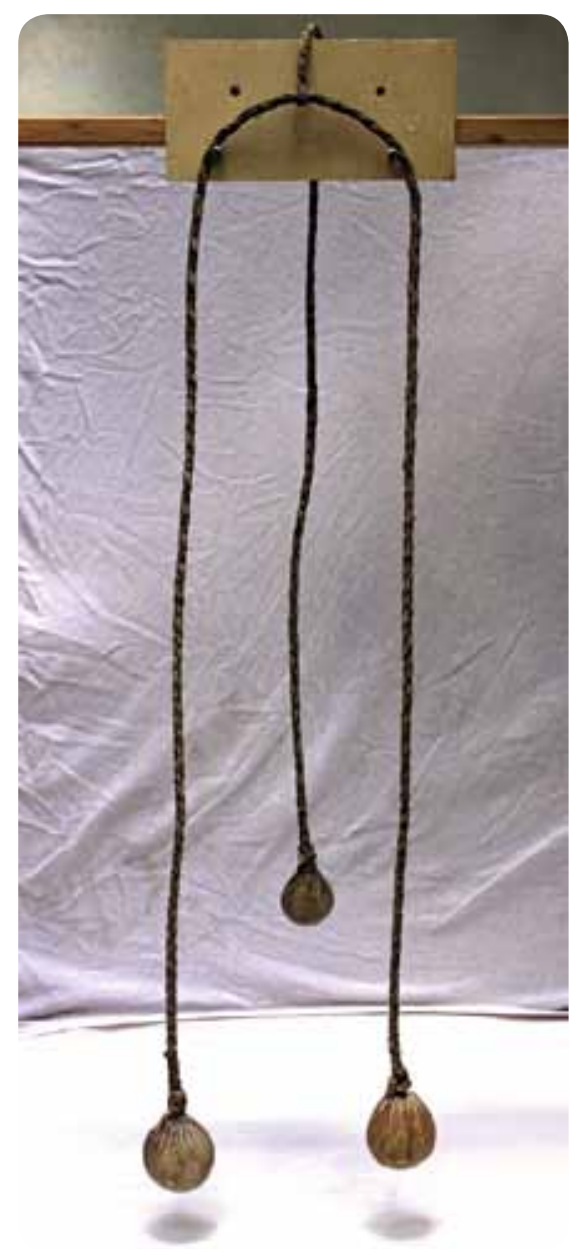

Abb. 1: Originalbola aus Argentinien
Speer, Bumerang und Tomahawk, Pfeil und Bogen und Armbrust).

In unserem Kulturkreis ist die Bola (span. Kugel) oder Boleadra weniger bekannt. Sie ist ein wirkungsvolles Wurfgerät und wurde ursprünglich von den Chinesen, den Eskimos und den indigenen Völkern Südamerikas benutzt. Die einfachste Bola, die Bola perdita (perdita $=$ die Verlorene) war eine sehr geschätzte Waffe im Wert eines halben Pferdes: Am Ende einer ca. 40 Zentimeter bis zwei Meter langen Schnur war ein kugelförmiger Gegenstand befestigt (z. B. ein Stein). Das Ende mit dem Gewicht wirbelte man über dem Kopf und schleuderte die Bola so in Richtung des zu treffenden Lebewesens (Wikipedia o. J.). Die heutigen Bolas haben wenigstens zwei Kugeln. Entweder werden sie in der Mitte der Schnur oder an einer der Kugeln gegriffen und durch kurzes Schwingen über dem Kopf Richtung Ziel geschleudert. Auf diese Art und Weise soll entweder ein Wild erlegt oder - so wie es die südamerikanischen Gauchos noch heute praktizieren - Vieh eingefangen werden. Die Bola wird da- bei so geworfen, dass sie sich um die Vorderbeine z.B. einer fliehenden Kuh wickelt, diese dadurch zusammenbindet und somit das Tier zu Fall bringt.

Abb. 1 zeigt eine argentinische Bola mit drei Kugeln, die aktuell von Gauchos benutzt wird. Die Bandlänge beträgt von Kugel zu Kugel $150 \mathrm{~cm}$ (120150 Gramm pro Kugel bei ca. $5 \mathrm{~cm} \varnothing$ ), wobei die Kugeln ummantelt sind mit einer Lederhülle. Verbunden sind sie mit einem geflochtenen Lederband, das aus drei Streifen (Breite jeweils $5 \mathrm{~mm}$ ) besteht. Die dritte Kugel hat lediglich einen Durchmesser von ca. $4 \mathrm{~cm}$ mit einem Gewicht von 60-80 Gramm und ist mit dem $75 \mathrm{~cm}$ langen Lederband locker in der Mitte des anderen Bandes befestigt.

\section{Die Bola als Sport- und Spielgeråt}

Auf der Grundlage der »Klassifikation von sportmotorischen Aufgaben « in Anlehnung an Leute und Stöber (2016) ist die Bola ein Sportgerät, das bewegt wird um ein Ziel zu treffen. Als Wurftech-

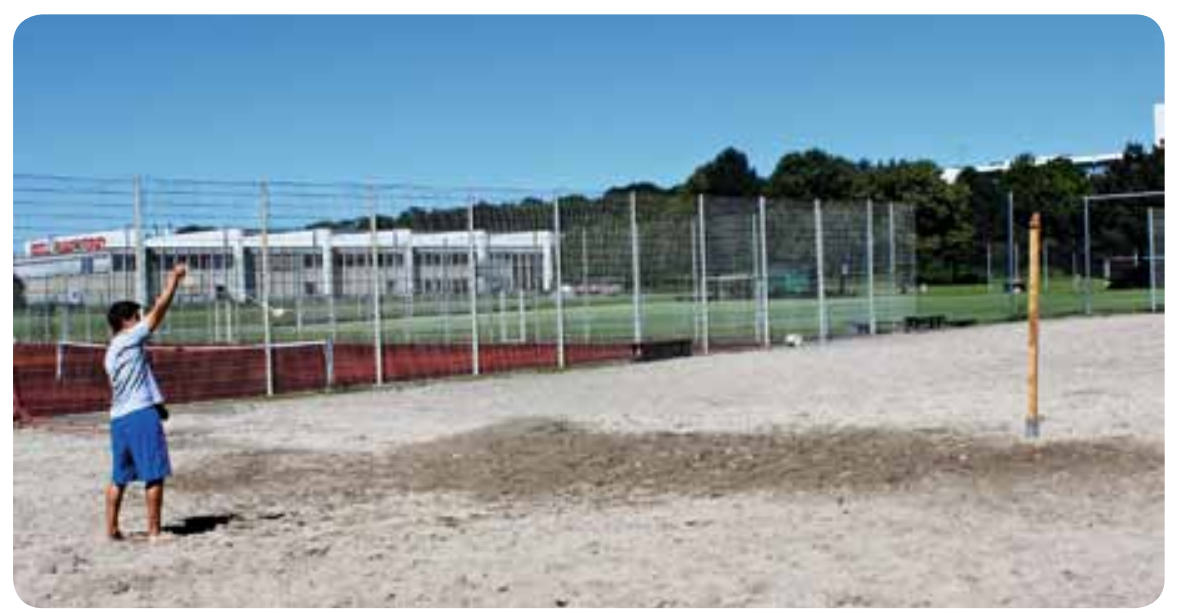

Abb. 2: Wurf auf einen Holzpfosten 


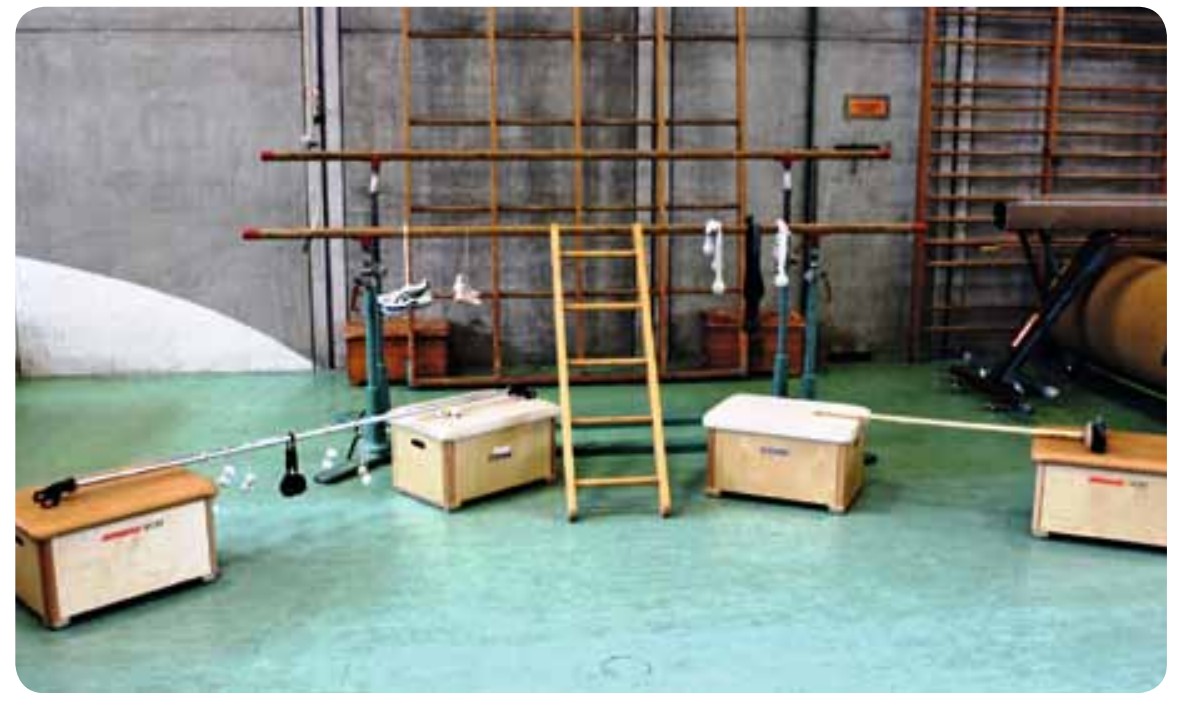

Abb. 3: Horizontale Ziele

nik wird dabei eine Drehbewegung bevorzugt, durch die der Gegenstand über dem Kopf rotiert wird, um ihn dann im richtigen Augenblick loszulassen, sodass er in Richtung Ziel (rotierend) fliegen kann (Abb. 2, horizontale Wurfausführung).

Möglich ist auch, dass der Wurfarm vertikal neben dem Körper die Bola zum Kreisen bringt. Auch hier ist zum richtigen Zeitpunkt loszulassen (vertikale Wurfausführung). Diese Technik bietet sich vor allem bei kurzen Bolas an. Es gibt nur wenige sportliche Aktivitäten, bei denen derartige Techniken genutzt werden, um ein Ziel zu treffen wie z.B. beim Gorodki (einem Wurfspiel) oder Lassowerfen, (Wagner 2010a). Die einfachste Aufgabenstellung wäre demnach, das Gerät zunächst möglichst weit zu schleudern. Etwas anspruchsvoller ist es hingegen, einen Gegenstand zu treffen (z.B. einen Kegel oder eine Dose auf einer Langbank). In Anlehnung an den Bola-Gebrauch der Gauchos könnte ein Gegenstand so getroffen werden, dass sich die Bola um diesen wickelt. Das könnte ein Volleyball-, Badminton- oder Reckpfosten sein. Aber auch Korbball- und Hochsprungständer sowie Kletterstangen könnten sich hierfür eignen. Im Freien wären freistehende Bäume, Geländer, transpor- tierbare Ständer und Torpfosten (Tor dann ohne Netz!) zu nutzen; ggf. könnten auch Speere fest in die Weitsprunggrube gesteckt werden. Auf Abb. 2 ist zu erkennen, wie ein ausgedienter Balken so bearbeitet wurde, dass er in die Fassung für einen Volleyballpfosten passt. (Die Bearbeitung und Gestaltung eignet sich - wie auch die Herstellung der Bolas - für einen Technik- oder Kunstunterricht oder als Projekt).
Abb.4: Einfache Bolas
Nicht nur das Werfen auf solche vertikal ausgerichteten Ziele ist reizvoll, sondern auch das Werfen auf horizontale: So kann sich die Bola um eine Reckstange und um einen Schwebebalken, um Kletterstangen und Torlatten sowie um Barrenholme oder Geländer wickeln. Der Kreativität bezüglich der Wurfziele sind keine Grenzen gesetzt: So kann beispielsweise ein Stufenbarren mit einer Reckstange oder eine fixierte Leiter mit drei bis fünf Sprossen (Leitergolf) als Wurfanlagen dienen (Abb.3).

\section{Die Fertigung einer Bola}

Bedauerlicherweise ist eine Bola in Deutschland (noch) nicht käuflich zu erwerben, so dass diese selbst zu fertigen ist: Auf der Grundlage der folgenden Systematik kann nun mit unterschiedlichen Gruppen (Kinder, Jugendliche, Erwachsene) und Bildungsabsichten (von der schnellen Improvisation bis zum anspruchsvollen Technikunterricht) eine funktionsfä-

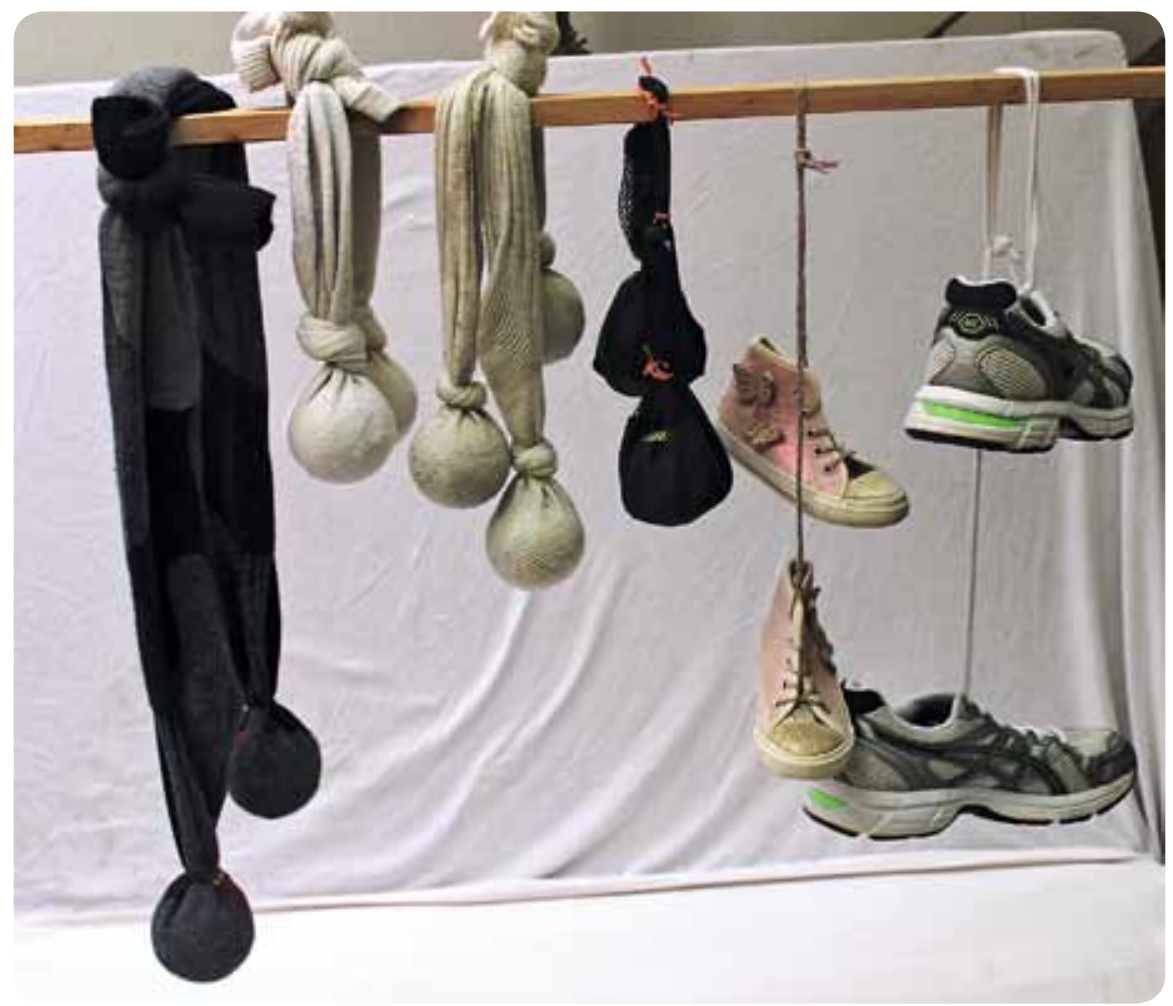


hige Bola hergestellt werden. Am einfachsten ist es, eine Bola entsprechend dem Original (Abb.1) nachzubauen. Sollen jedoch Eigenkreationen entstehen (idealerweise mit Abfall bzw. Alltagsmaterialien; Wagner 2009), wird eine genaue Analyse des Wurfgerätes notwendig. Bei einer solchen Analyse haben wir uns an der Idee des sogenannten "Morphologischen Kastens" orientiert (z. B. Zwicky 1966). Diese besagt, dass das Problem (hier die Bola) in sinnvolle Komponenten zu zerlegen sei, um entsprechend viele geeignete Lösungen zu entwickeln. Das Zusammenfügen der jeweils besten Lösung soll ein zufriedenstellendes Produkt ergeben.

Ausgehend von einer Originalbola (Abb.1) kann demnach das Wurfgerät in drei Teile zerlegt werden: In den kugelförmigen Gegenstand (Gewicht), in dessen Verpackung und in die Verbindung von Gewicht zu Gewicht. Als kugelförmiger Gegenstand könnte beispielsweise ein Golf-, Tennis- und Hockeyball, eine selbstgefertigte Holzkugel, ein Stein oder ein Schuh dienen. Eine Verbindung von Gewicht zu

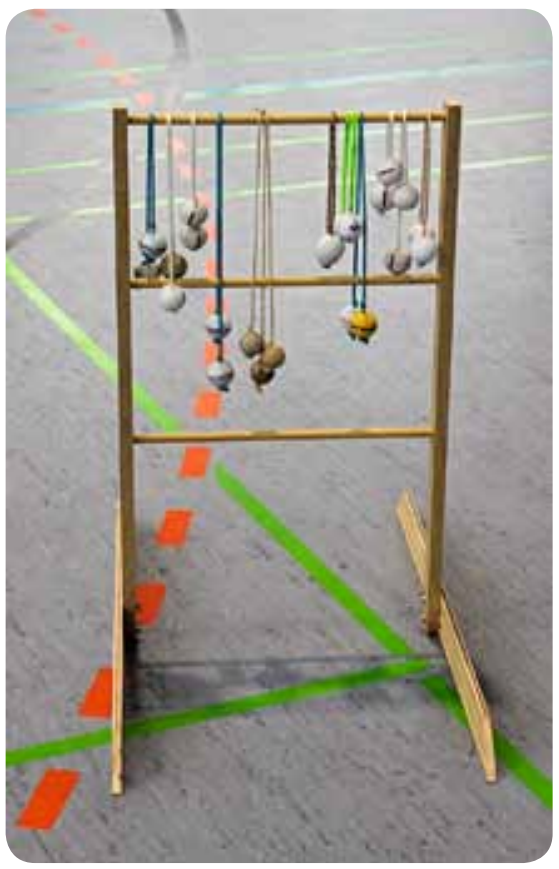

Abb. 5: Leitergolf mit Golfbolas unterschiedlicher Länge
Gewicht könnte z.B. mit einer dicken Schnur, einem Kevlarseil vom Volleyballnetz, Paracord, mit den Beinteilen von Kniestrümpfen oder mit Schnürsenkeln hergestellt werden. Als Verpackung des Gewichtes können z.B. das Fußteil von Kniestrümpfen, Schwitztuchbeutel oder selbstgefertigte Lederbeutel dienen. Werden nun die verschiedenen Lösungen der jeweiligen Elemente kombiniert, entstehen interessante Ergebnisse:

Eine Schuhbola (Abb.4, die beiden Bolas rechts) entsteht, indem zwei (drei) Sportschuhe mit möglichst großem Abstand von Schuh zu Schuh zusammengebunden werden. Dieser Abstand kann ggf. mit einer Zwischenschnur vergrößert werden.

Eine Strumpfbola (Abb.4, die drei Bolas links) entsteht, indem ein Tennisball jeweils in das Fußteil eines Kniestumpfes gesteckt wird. Danach wird das Fußteil so verknotet, dass der Ball stramm im Zehenbereich steckt. Es ist auch möglich, das Fußteil mit einer Schnur abzubinden. Die beiden Ballstrümpfe werden nun so, wie bei der Schuhbola, mit möglichst großem Abstand zusammengeknotet. Die ZweierBola kann mit einem weiteren Ballstrumpf ergänzt werden.

Für die Schwitzbola (Abb.4, die vierte Bola von links) werden zwei bzw. drei netzartige Schwitztuchverpackungen benötigt, in die jeweils ein Tennisball gesteckt und mit den eigenen Bändern so, wie bei der Schuhbola, zusammengebunden werden. Zur Stabilisation des Balles ist dieser, wie bei der Strumpfbola, abzubinden. Da Schwitztuchverpackungen im Regelfall wenig Stabilität aufweisen, sollte kein Hockeyball verwendet werden.

Für die Golfbola (Abb.5) werden zwei (oder drei) Golfbälle benötigt, die entweder z.B. mit einer Schnur oder mit einem Kevlarseil ( $\varnothing 4 \mathrm{~mm}$ ) verbunden werden. Die Bälle werden mit-

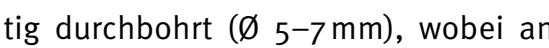
einem Ende der Bohrung das Loch so zu vergrößern ist, dass der Knoten de Schnur, der nach dem Durchführen des Seiles am Ende des Seiles angebracht wurde, in der Vertiefung verschwindet. Mit Heißkleber sollte der Knoten in der Vertiefung festgeklebt werden. Bei dieser Form der Bola ist ein Abstand von $60-80 \mathrm{~cm}$ von Kugel zu Kugel ausreichend. Wird die Verbindung länger, sind die Golfbälle zu leicht.

Will man eine authentische Bola herstellen (d.h., Orientierung an Gewicht und Maßen), könnte eine Hockeybola, eine Holzbola oder eine Steinbola mit Kevlarseil, Schnur oder Paracord entstehen.

Für die Hockeybola (Abb.6, links) werden drei Hockeybälle, eine ca. $190 \mathrm{~cm}$ lange und eine $90 \mathrm{~cm}$ lange Verbindung benötigt. Die Herstellung entspricht der der Golfbola (Abb. 5).

Für die Holzbola (Abb. 6, Mitte) werden drei Hartholzkugeln, etwa in der Größe von Hockeybällen, benötigt. Sollen die Kugeln selbst gefertigt werden, reicht es aus, lediglich die Ecken und Kanten eines Holzwürfels abzurunden. Die gebohrten Kugeln können z.B. auch mit drei Kevlarseilen verbunden werden, die Verbindung zwischen Seil und Holz entspricht der der Golfbola (Abb. 5).

Für die Steinbola (Abb.6, rechts) werden drei eckige Steine mit den MaBen $40 \times 20 \times 30 \mathrm{~mm}$ und zwei kräftige Schnüre benötigt. Jeweils an das Ende der ca. $260 \mathrm{~cm}$ langen Schnur wird ein Stein mit einem selbstzuziehenden Knoten in Form eines Päckchens gebunden. An das kurze Stück $(130 \mathrm{~cm})$ wird analog ein Stein gebunden, wobei dieses Seil genau in der Mitte des langen Seiles festgemacht wird. Dieser Bauvorschlag (Steinbola) sollte nur mit verantwortungsbewussten Jugendlichen oder Erwachsenen realisiert werden. Vor allem sollte das Verpacken der 


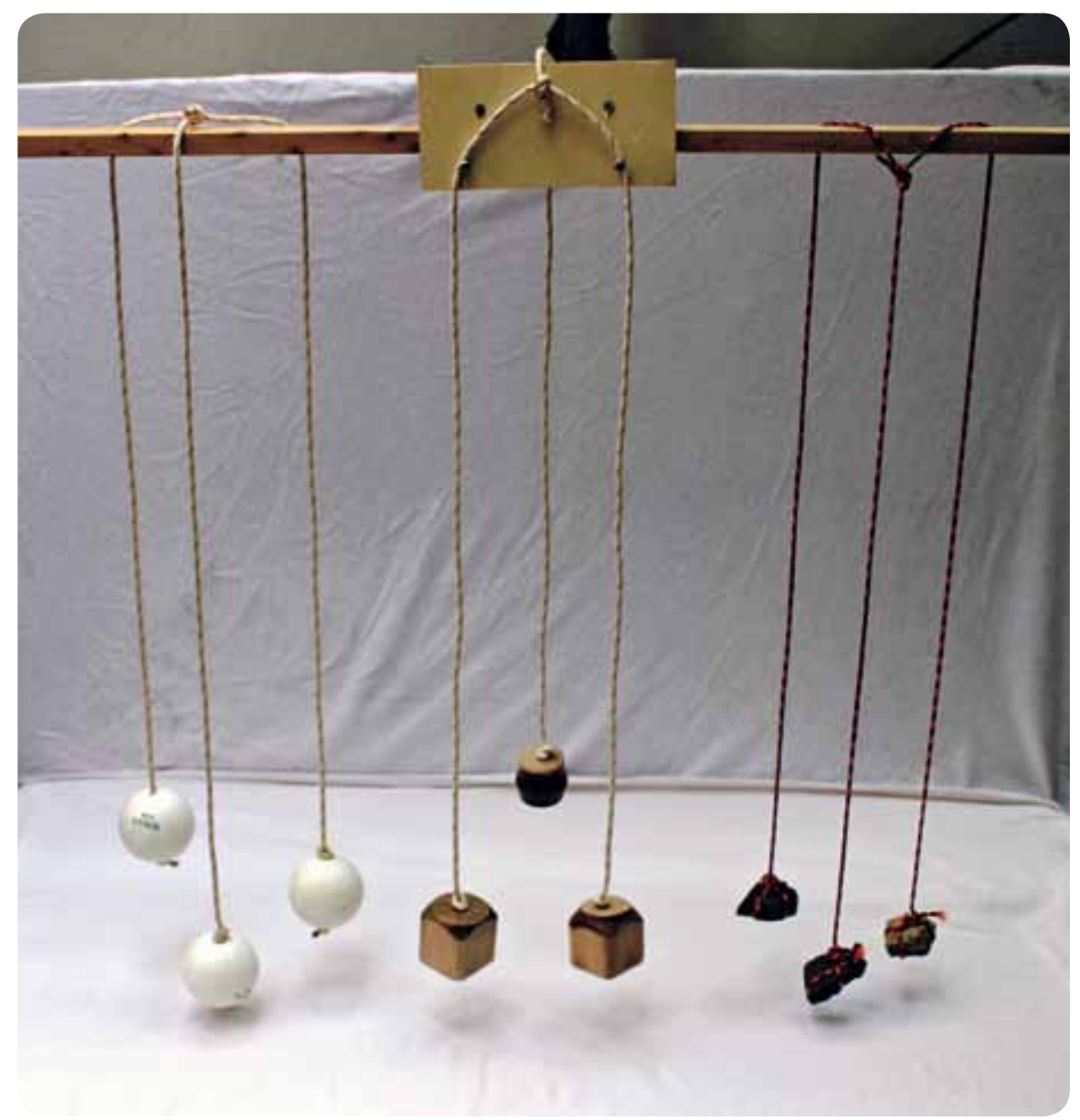

Abb. 6: Kugel-, Holz- und Steinbola

Steine mit äußerster Sorgfalt und Präzision erfolgen, sodass sich beim Werfen kein Stein aus der Verpackung lösen kann. Auch das Werfen muss unbedingt unter strengen Sicherheitsmaßnahmen und ausschließlich im Freien erfolgen. Auch sollte geprüft werden, ob die Steinbola beim Treffen von Gegenständen diese nicht beschädigen kann (z. B. Bäume).

Zum Werfen der Varianten Schuh-, Strumpf-, Schwitz- und Golfbola werden die Bolas an den Kugeln gegriffen. Bei den Varianten der Hockey-, Holz- und Steinbola greift man zum Werfen in der Mitte der Schnur (Abb. 2).

Es bietet sich an, das Thema offen, z.B. mit Orientierung an dem genetischen Lernen, einzuführen (Wagner 2010b). So könnten die Kinder, Jugendlichen oder Erwachsenen - nach dem Vorstellen der Bola mit ihrem Zweck - eigene Spielgeräte, Ziele und Wurftechniken entwickeln.

\section{Hans-Jürgen Wagner}

DOI 10.2378 / motorik2017.arto6d

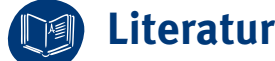

Leuchte, S., Stöber, K. (2016): Ordnung in der Vielfalt - Klassifikation sportmotorischer Aufgaben. Dr. Kovac, Hamburg

Wagner, H.-J. (2009): Das neue Denken - Das Neue denken ... Wie Alltagsgegenstände und Abfallmaterialien für Sport, Spiel und Bewegung genutzt werden können. motorik 32 (2), 82-87 Wagner, H.-J. (2010a): Low tech statt high tech. Spielgeräte selber bauen. Werfen wie die Leute aus dem Norden. Teil 2: Gorodki. SportPraxis $51(9+10)$, 23-27

Wagner, H.-J. (2010b): Genetisches Lernen als Möglichkeit zur Vermittlung des Volleyballspiels in der (Grund) schule. In: Langolf, K., Roth R. (Hrsg.): Volleyball international in Forschung und Lehre. Czwalina, Ahrensburg, 103-116.

Wikipedia (o. J.): Bola.https://de.wikipedia. org/wiki/Bola, 20.09.2016

Zwicky, F. (1966): Entdecken, Erfinden, Forschen im morphologischen Weltbild. Droemer und Knaur, Zürich / München 